\title{
Towards Automatic Systems Architecting
}

From abstract concept to architecture

Gustavo Pinheiro, Felipe Simon and Geilson Loureiro July, 2007
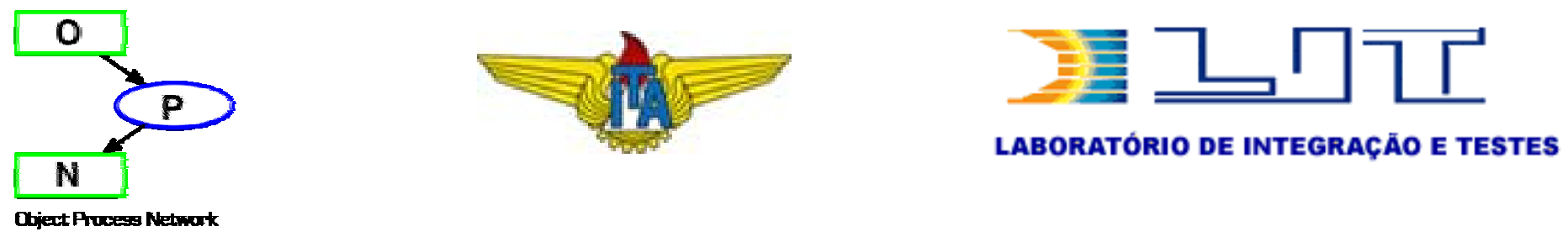


\section{Agenda}

- Intro to the Project

- The Current Approach vs. The New Approach

- Applications

- The Hierarchical OPN

- Further Development / Conclusion 


\section{Introduction}

- Systems Architecture Domain

- To Consider many options when designing a system

- To be able to model a specific solution/architecture in an efficient manner

- Available Languages / Tools :

- OPM, Structured Analysis, UML, SysML ...

- OPN (Decision-Support tool)

- Goal:

- To define a new approach that allows both perspectives to be considered 


\section{The Current Approach}

- Currently, decision-support tools are completely separated from system architecture modeling tools:

- When deciding: you do not have instruments for a common visual understanding of the system

- When modeling: you do not have instruments for deciding which way to move forward

- OPM Model:

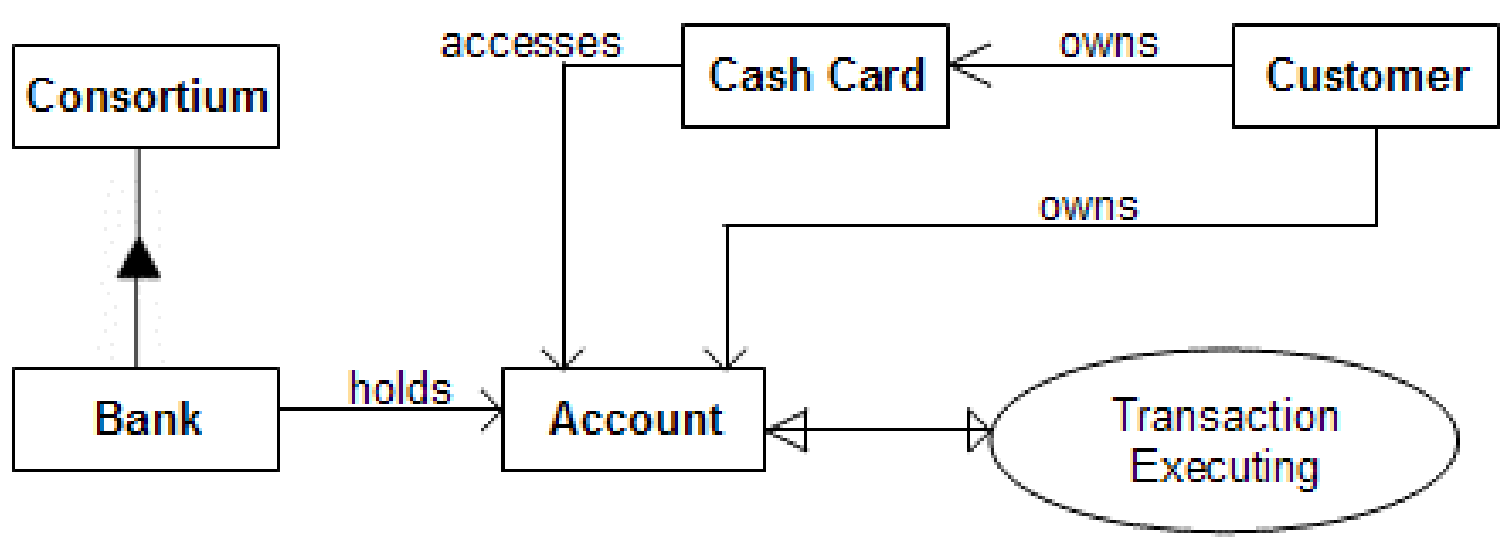




\section{The Current Approach}

- OPN Graph (Higher-Lever of Abstraction) :

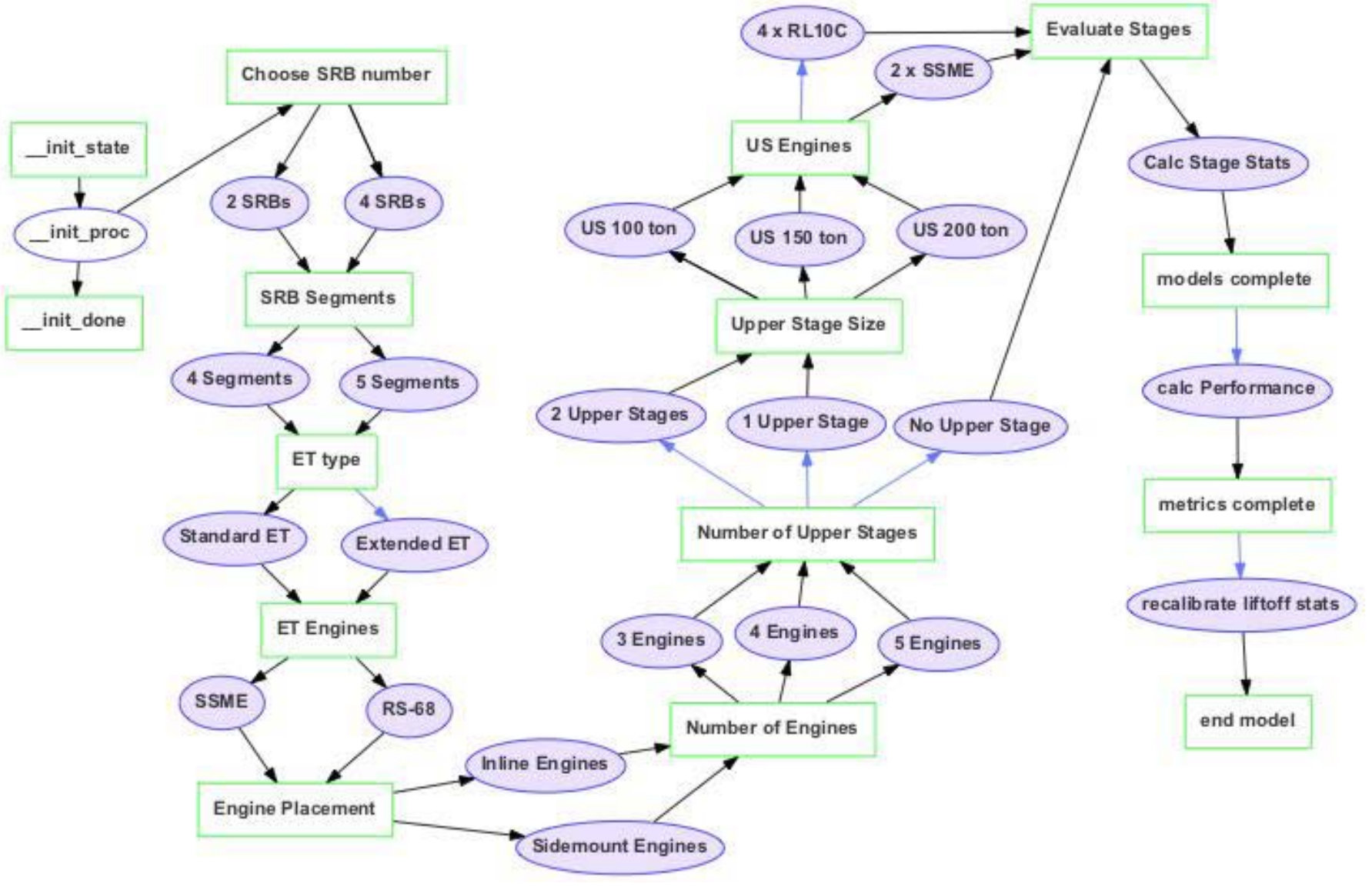




\section{The New Approach}

- Association OPM-OPN

- Model the Space of Options with OPM

- Systematic Translation to OPN

- Results presented using OPM notation (for each architecture)

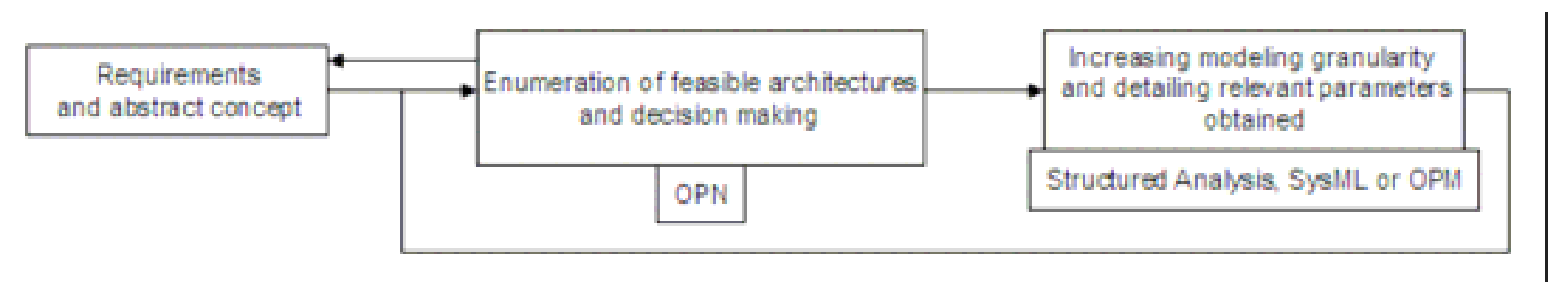

- That's what we call "Automatic Systems Architecting" 


\section{The New Approach - Step-by-Step}

1. Define the function to be performed by your system.

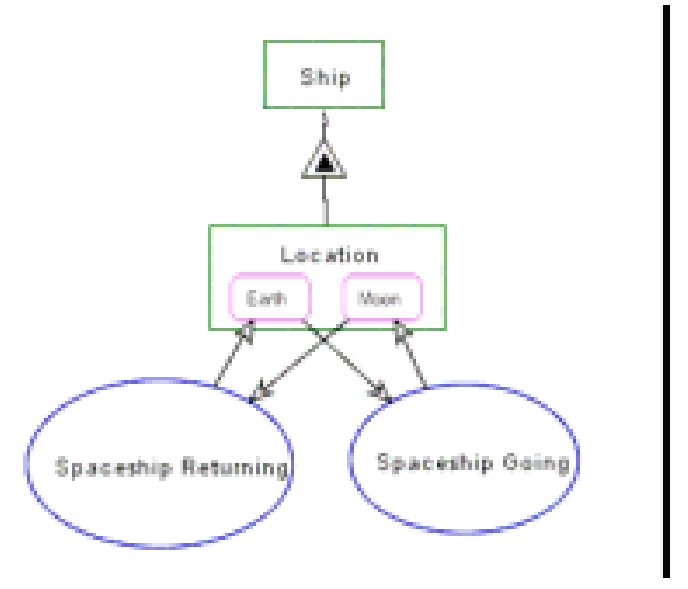

2. Define Boundary Conditions (BC) to your Problem (they hinder the change from an initial state to a final state).

3. Assign Functions that "solve" these boundary conditions and the parameters which are important for making a decision to "feed" the OPN model.

4. Show Specialization possibilities for this solution and how they alter the parameter's values.

5. If necessary, define New $B C$ to these functions (iterate between 2, 3 and 4).

6. Check Architectures selected by OPN 


\section{Application 1}

- Market of Sodas - Logistic issues

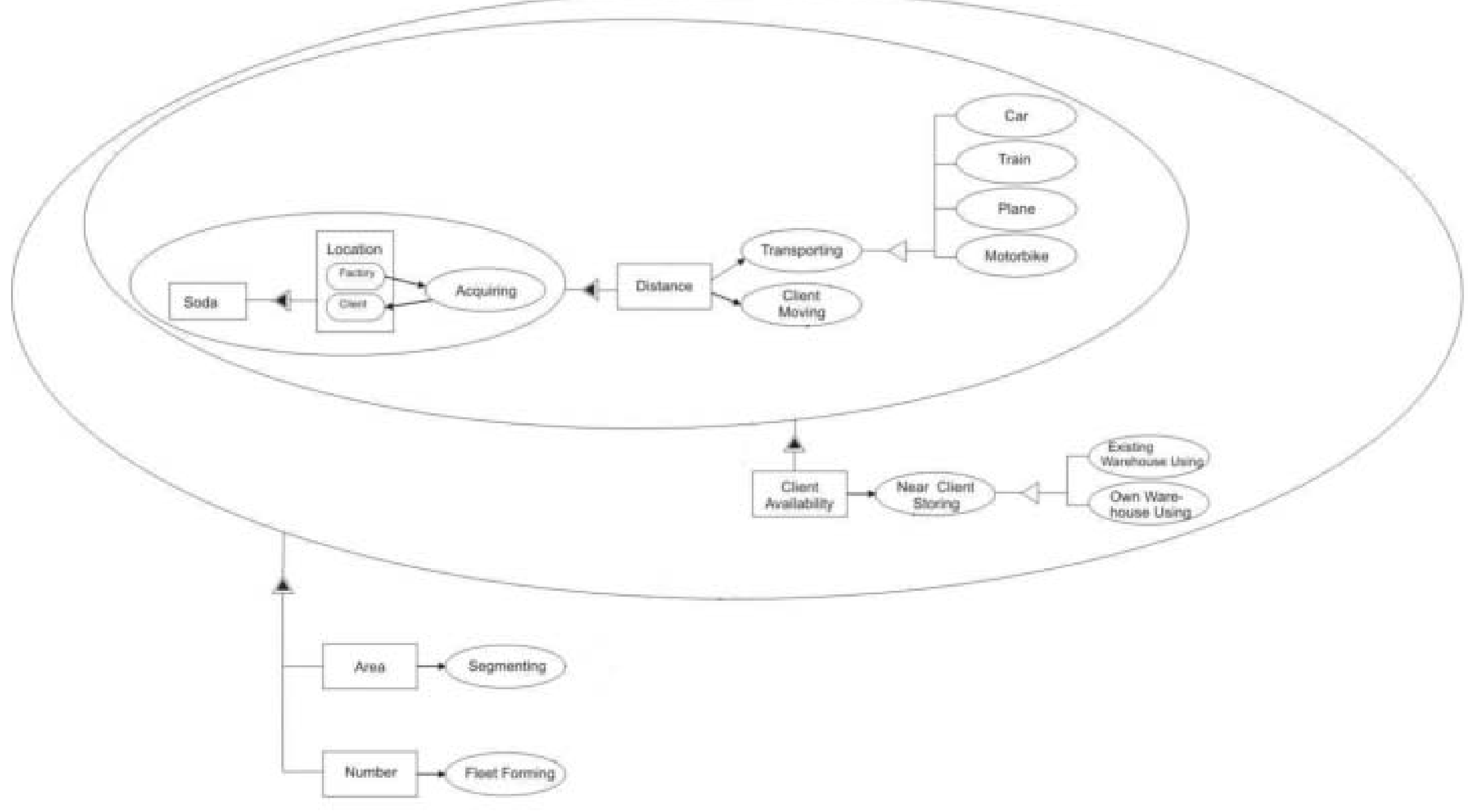


Application 1

- Market of Sodas - Logistic issues

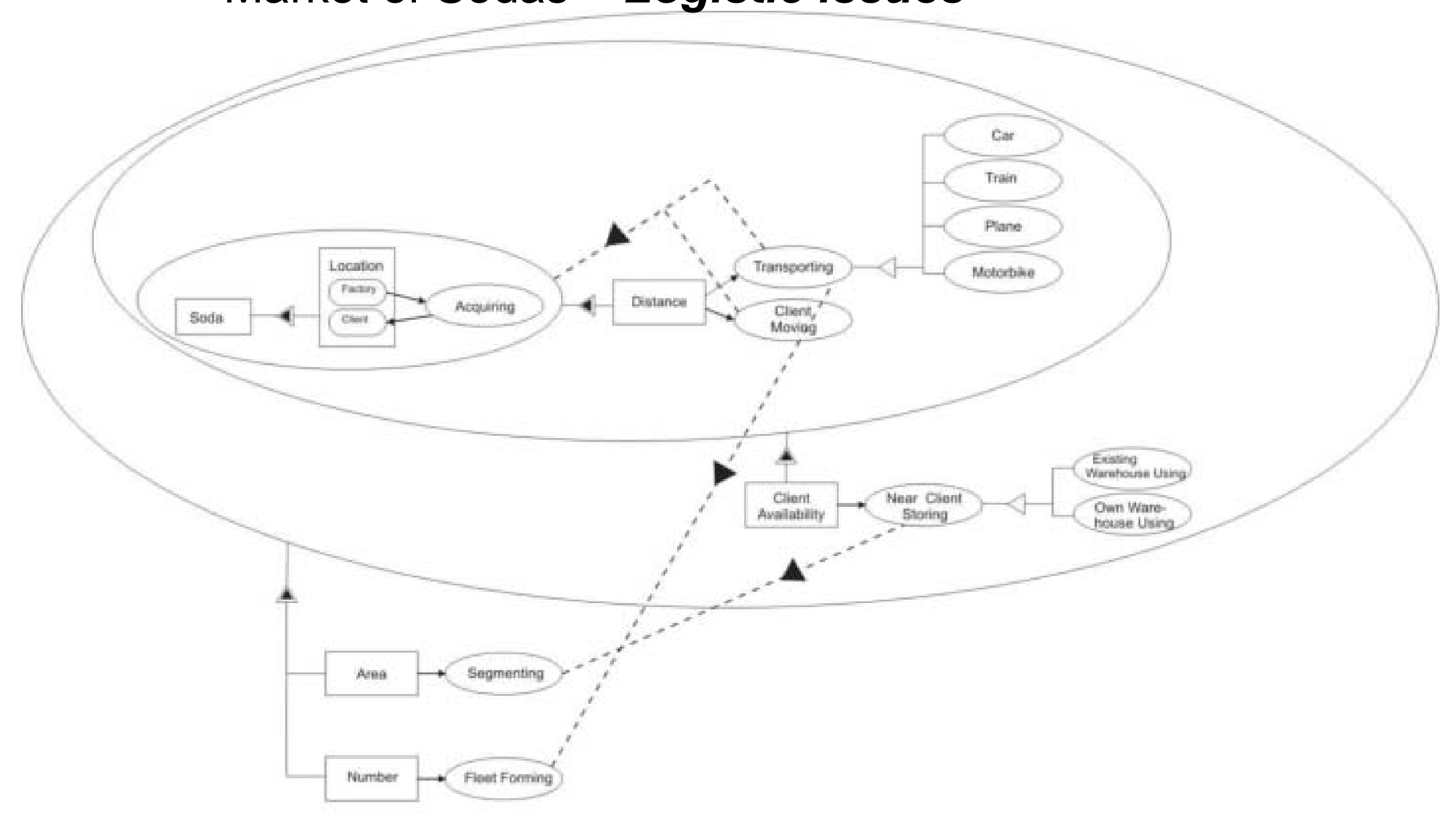




\section{Application 1}

- Adding parameters that will feed the OPN model

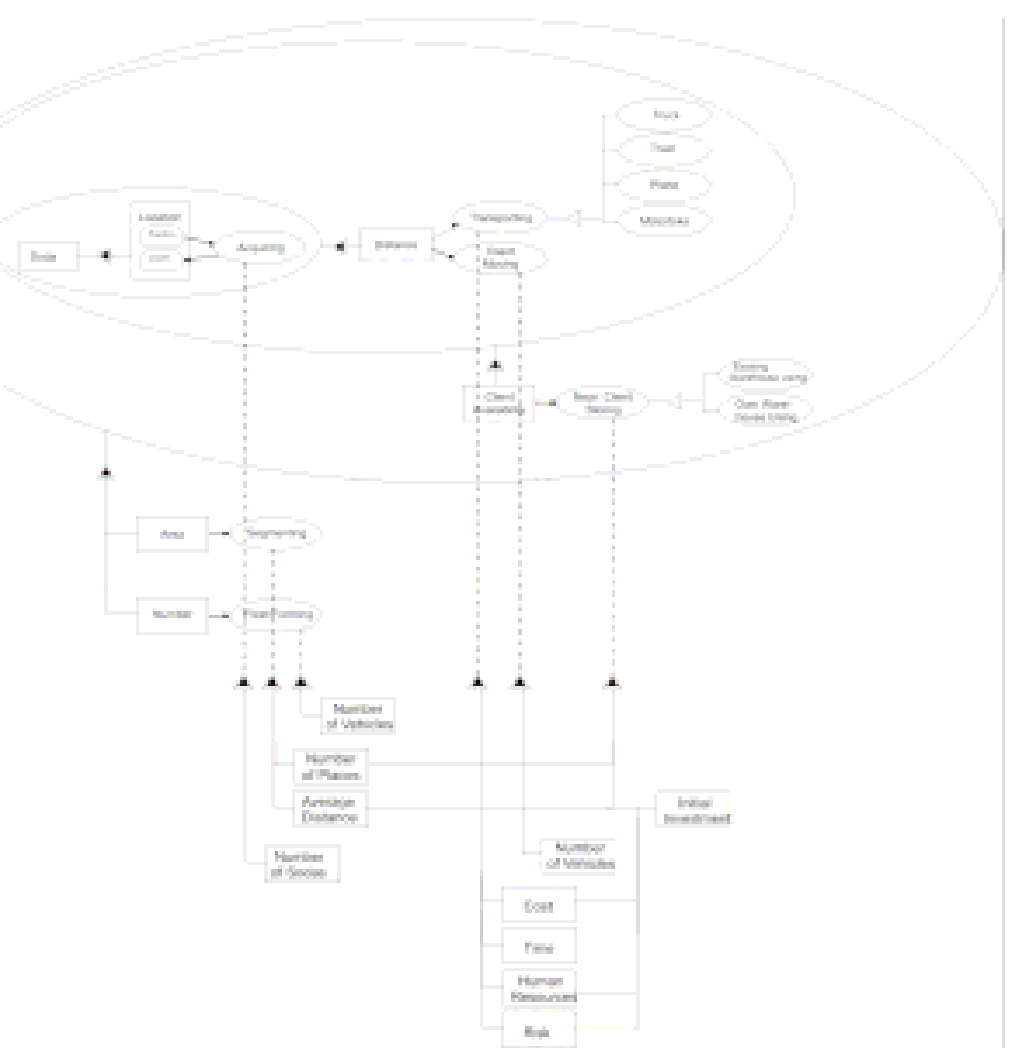

The functions are "described" in terms of these parameters 


\section{Application 1}

- Translating the decisions to be made to OPN

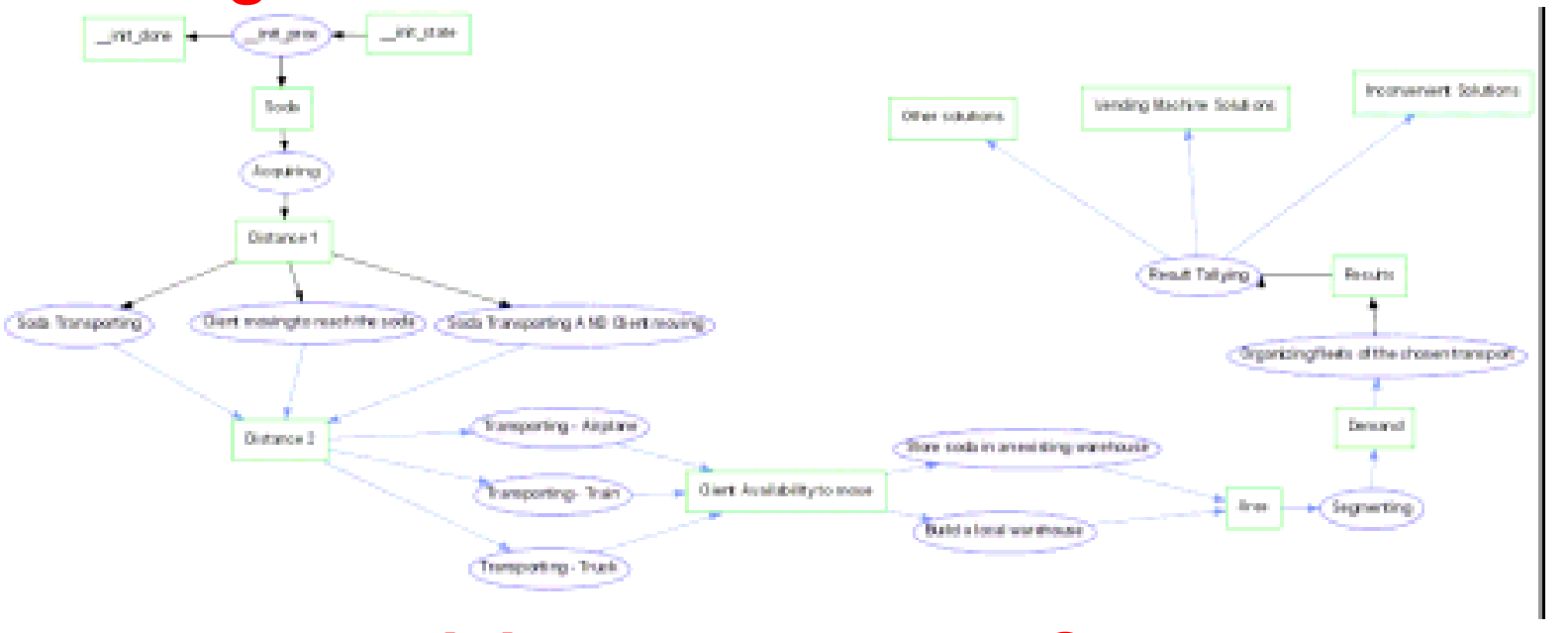

- Modeling the decisions made using OPM notation 


\section{Application 1}

- Eventually, the decisions made can be modeled in the form of the actual system with OPM

(This OPM model represents an architecture pointed out by OPN)

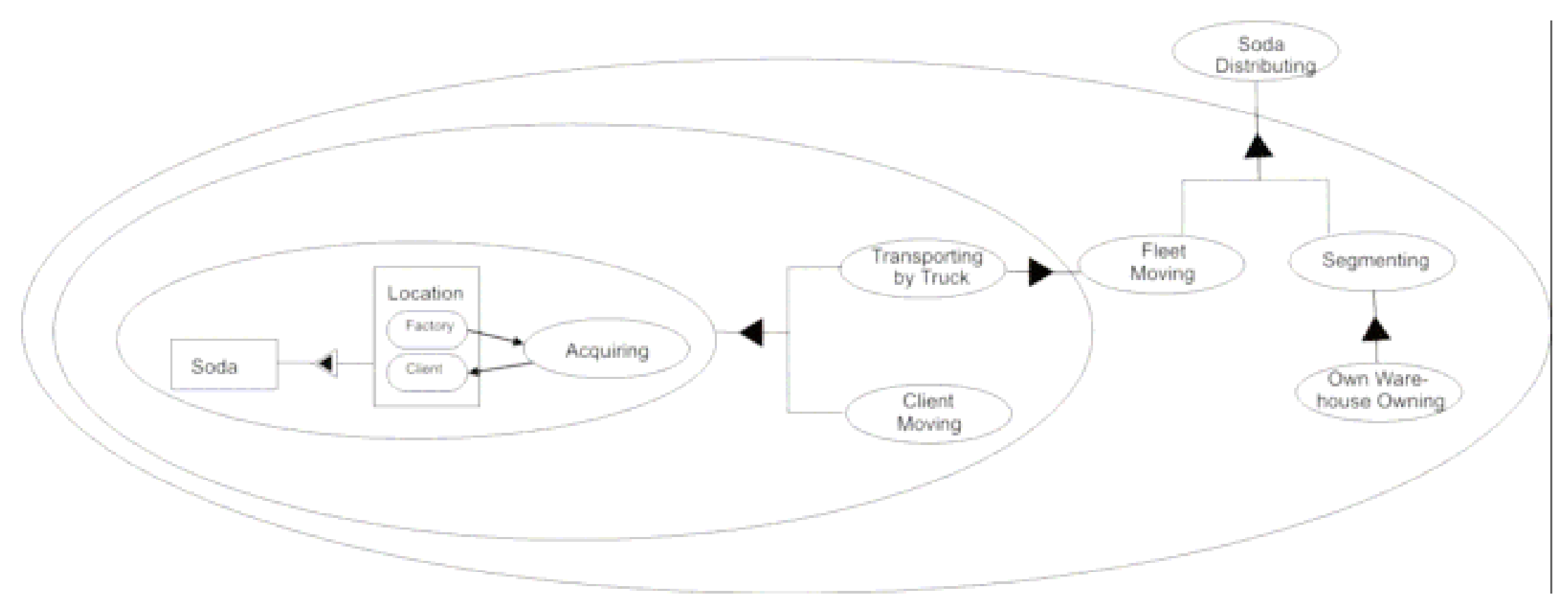




\section{Applications - Questions}

- For a simple application, a single OPN model may be sufficient to model all the "points of decision" to be considered.

- The more complex our system becomes, the more difficult it is to integrate knowledge from different domains (i.e. decisions in different areas) in a single OPN model.

- To try to model such a system using OPN current features leads to a model with low cohesion and high coupling.

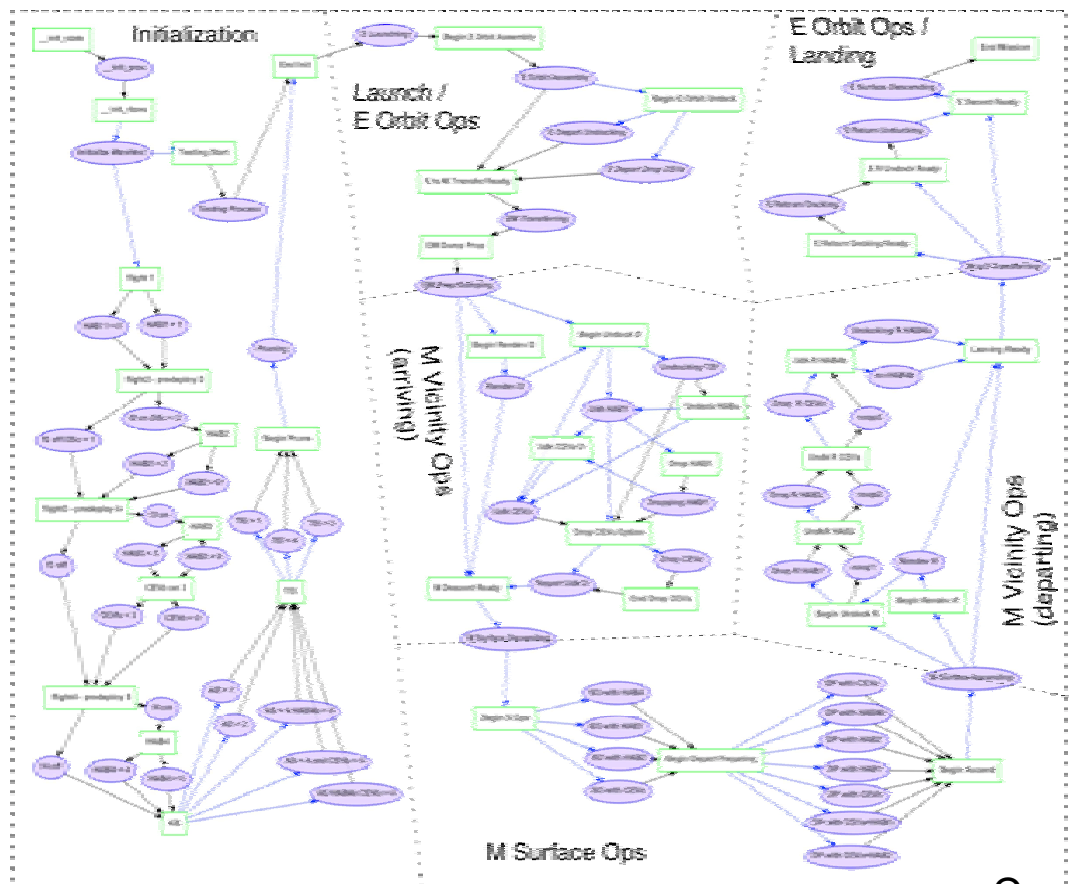




\section{The Hierarchical OPN}

- But what we need is high cohesion and low coupling!

- Solution: The Hierarchical OPN

- The recursivity presented in the new approach would be defined in lower levels OPN models.

- At higher levels, complexity would be hidden.

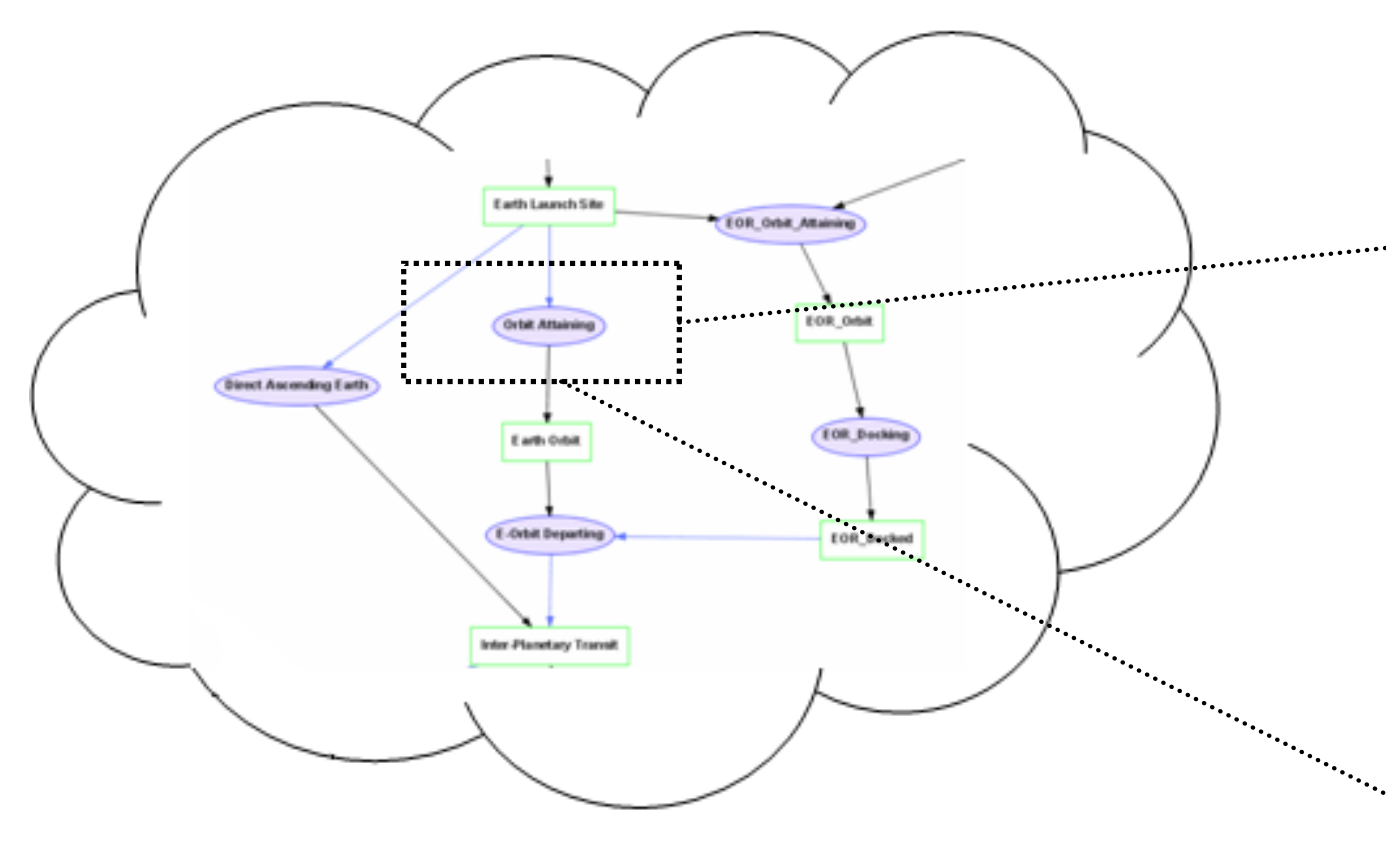

Higher-level

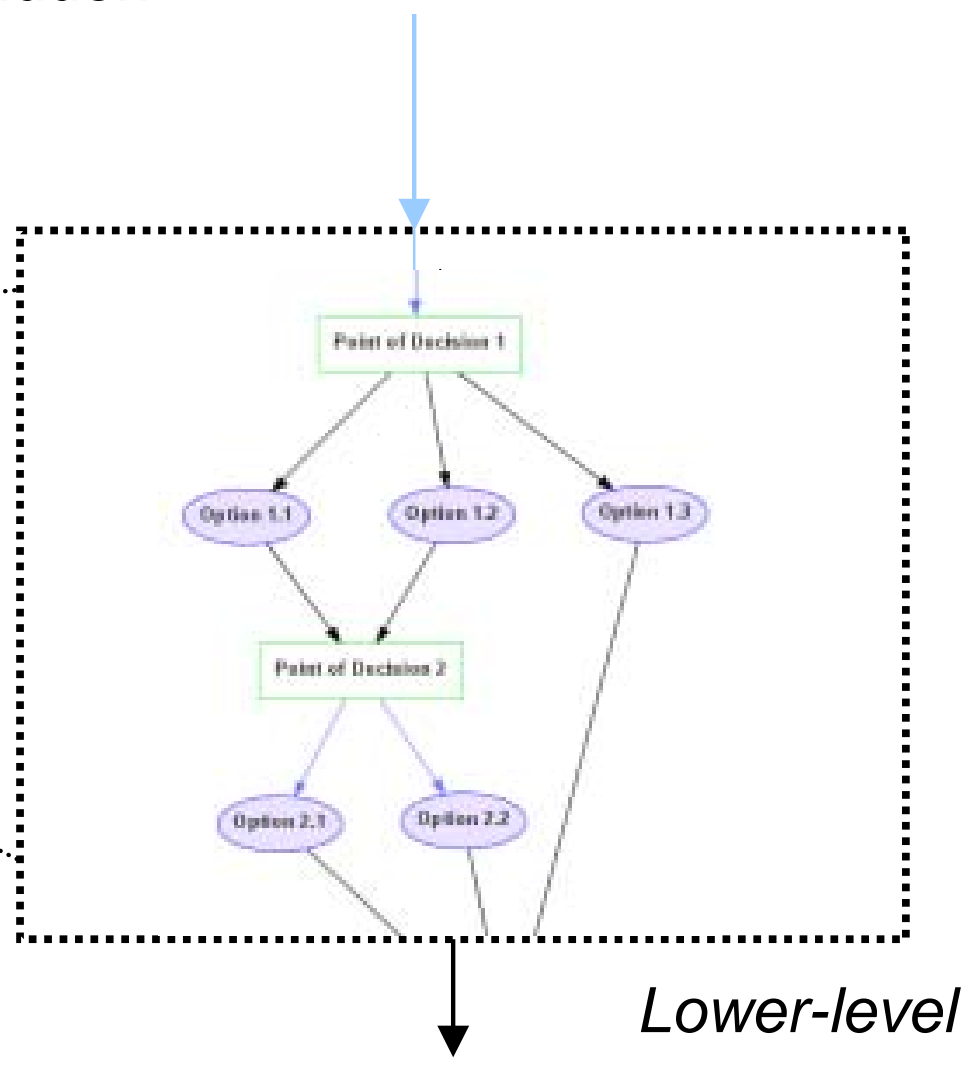




\section{The Hierarchical OPN}

- Pros:

- To allow the design of complex systems (that involves experts from multiple domains) using OPN tool.

- To provide higher cohesion and lower coupling

- The New Approach:

- Tool that integrates OPM with Hierarchical OPN

- Note: When different notations are been used, one should to translate all of them to OPM. We've verified it's quite easy translation between SA, OPM, SysML, UML 


\section{Application 2}

- Lunar Lander (Under Development)

- This example will show:

- Exactly how the different levels will communicate with each other during simulation process; (parameters from higher levels modifying lower levels parameters and vice-versa).

- That this "better organized" approach (high cohesion and low coupling) will lead to model reuse
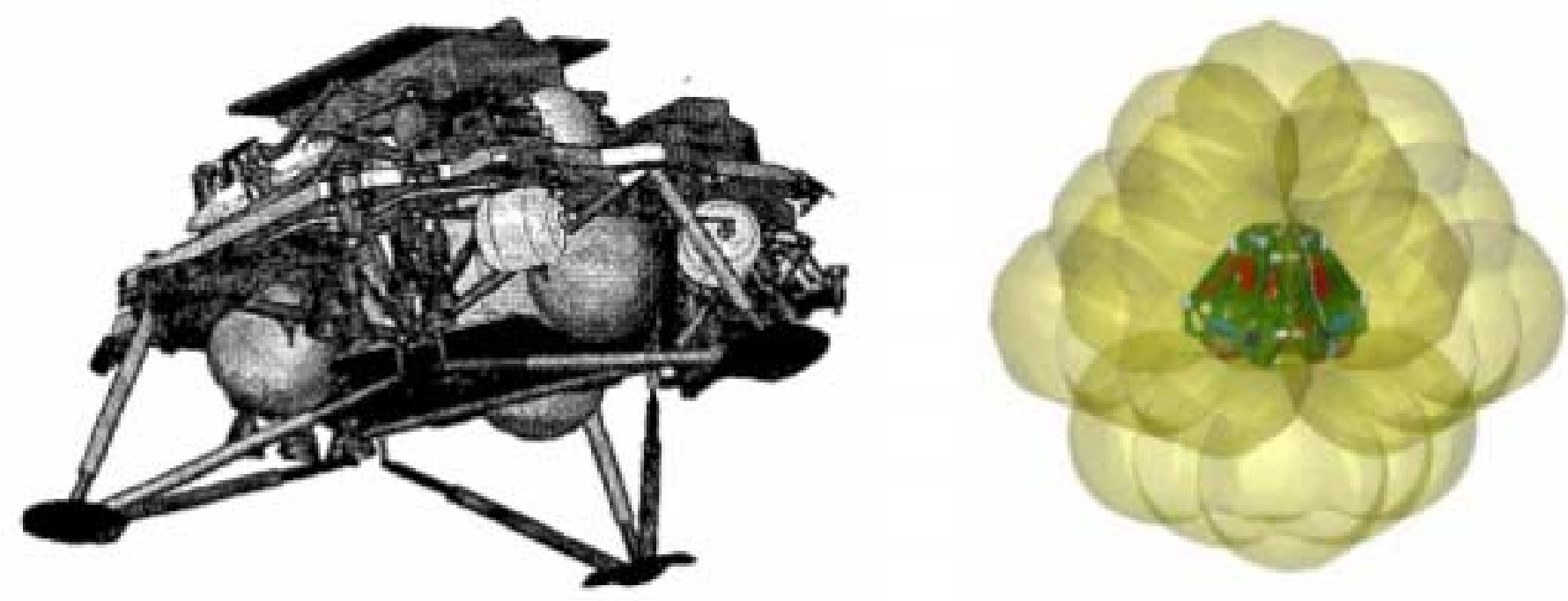

Legged and Air-bag concepts - Two of the options considered for the Lunar Lander 


\section{Further Development / Conclusions}

- How to figure out to which extent we should model? We could spend effort modeling a solution that will never be developed!

- How can the models evolve?

- Conclusion of current study case (Lunar Lander)

- Implementation of an user-friendly tool able to mechanize the proposed approach (Association OPM + Hierarchical OPN) 